\title{
Endoscopic vacuum-assisted closure system (E-VAC): case report and review of the literature
}

\author{
Maciej Borejsza-Wysocki, Krzysztof Szmyt, Adam Bobkiewicz, Stanisław Malinger, Józef Świrkowicz, \\ Jacek Hermann, Michał Drews, Tomasz Banasiewicz \\ Department of General and Endocrine Surgery and Gastrointestinal Oncology, Poznan University of Medical Sciences, Poznan, Poland
}

Videosurgery Miniinv 2015; 10 (2): 299-310

DOI: $10.5114 /$ wiitm.2015.52080

\begin{abstract}
Negative pressure wound therapy (NPWT) has become a standard in the treatment of chronic and difficult healing wounds. Negative pressure wound therapy is applied to the wound via a special vacuum-sealed sponge. Nowadays, the endoscopic vacuum-assisted wound closure system (E-VAC) has been proven to be an important alternative in patients with upper and lower intestinal leakage not responding to standard endoscopic and/or surgical treatment procedures. Endoscopic vacuum-assisted wound closure system provides perfect wound drainage and closure of various kinds of defect and promotes tissue granulation. Our experience has shown that E-VAC may significantly improve the morbidity and mortality rate. Moreover, E-VAC may be useful in a multidisciplinary approach - from upper gastrointestinal to rectal surgery complications. On the other hand, major limitations of the E-VAC system are the necessity of repeated endoscopic interventions and constant presence of well-trained staff. Further, large-cohort studies need to be performed to establish the applicability and effectiveness of E-VAC before routine widespread use can be recommended.
\end{abstract}

Key words: endoscopic vacuum-assisted wound closure system, Endo-Sponge, vacuum-assisted wound closure therapy, eosophageal leakage, rectal anastomotic leakage, pancreatico-gastric anastomotic insufficiency.

\section{Introduction}

Negative pressure wound therapy (NPWT) is a well-established method of treatment of complicated and chronic wounds. The negative pressure is applied to the wound via a special vacuum-sealed sponge. This sponge continuously or intermittent removes wound secretion or edema, improves microcirculation and accelerates formation of granulation tissue. Since its introduction in the late 1990s, the number of indications for the VAC system has constantly increased [1].

Endoscopic vacuum-assisted wound closure system (E-VAC) therapy has been proven to be an important alternative in patients with upper and lower intestinal leakage not responding to standard endoscopic and/or surgical treatment procedures. The method has been adapted from NPWT for complicated ulcer and postoperative wounds [2, 3]. Recent studies have reported the applicability of an intracavitary and endoluminal vacuum-assisted wound closure system to close rectal anastomotic fistulas and to treat anastomotic leaks after esophagus resection $[4,5]$.

Intrathoracic spontaneous or postoperative anastomotic leakages are considered to be potentially life-threatening conditions due to the possibility of mediastinitis development and consecutive sepsis [6]. The reported incidence of esophageal anasto-

\section{Address for correspondence}

Krzysztof Szmyt MD, Department of General and Endocrine Surgery and Gastrointestinal Oncology, Poznan University of Medical

Sciences, 49 Przybyszewskiego St, 60-355 Poznan, Poland, phone: +48 507066 108, e-mail: krzysztof.szmyt@gmail.com 
motic leaks after surgery ranges from 1\% to $30 \%$ [7]. Endoscopic interventions including application of metal clips, fibrin glue, and placement of self-expanding metal or plastic stents (SEMS/SEPS) have been reported to achieve successful closure of postoperative anastomotic leaks. The new, promising and alternative method is an endoscopic insertion of sponges into the cavity induced by the leak (intracavitary) or intraluminal insertion directly onto the defect.

One of the major complications after rectal surgery is anastomotic leakage, with an incidence rate of up to $30 \%$ [8]. This complication mostly occurs in patients after neoadjuvant radiochemotherapy with deep rectal anastomoses [9-11]. The presence of rectal anastomotic leakages significantly increases the morbidity and mortality, despite potentially successful surgery. Antibiotics, transrectal rinsing and drainage have been established treatment ways for patients without peritonitis. If sepsis is uncontrolled, a surgical procedure with debridement and stoma creation are needed.

The aim of this article is to analyze and summarize the available literature on the applicability of E-VAC in upper gastrointestinal, rectal and pancreatic surgery complications, mainly associated with the presence of anastomotic leakage or perforation. Advantages and limitations of this technique are also discussed.

\section{Case report}

A 72-year-old patient was admitted to the Department of General, Endocrinological and Gastroenterological Oncology Surgery in Poznan, because of a diagnosed tumor in the head of the pancreas. The lesion had been revealed 3 months earlier in ultrasonography examination due to non-specific abdominal pain. The patient suffered from chronic obstructive pulmonary disease (COPD) and primary arterial hypertension as comorbidities. According to the physical examination a giant pathologic mass in the epigastric region was palpable. A computed tomography (CT) scan revealed a tumor in the head of the pancreas measuring $14 \times 11 \times 10 \mathrm{~cm}$. A movable tumor, rich in pathologic vascularity, without any signs of infiltration of other organs, was detected in the head of the pancreas. There were no other pathologies in the abdominal cavity. Pancreatoduodenectomy (Traverso procedure) was performed.
Intraoperative histopathological examination diagnosed a neuroendocrine tumor of the pancreas.

At day 6 following the primary surgery a pancreatico-gastric anastomotic insufficiency was diagnosed. An increased level of amylase (108 $000 \mathrm{U} / \mathrm{ml})$ was detected in the examination of fluid collection from the abdominal cavity. The patient was re-operated twice, at days 7 and 15 after the primary surgery. Revision of the insufficient anastomosis was performed. In the first surgery, sutures were added to the insufficient anastomosis. The re-operation was unsuccessful; leakage was observed again from the second day after the reoperation. In the second re-operation massive inflammation in the upper abdomen was observed and only the area of anastomotic dehiscence was drained. Total parenteral nutrition and somatostatin management were implemented. Because of deterioration of the patient's general condition confirmed by biochemical markers as well as increased fluid contents from drainage, the patient was qualified for E-VAC therapy at day 20 after the primary surgery (Photo 1 ).

Gastroscopy in sedation (without intubation) was performed, and anastomotic dehiscence was easily found. At the bottom of the fistula, one of the abdominal drains was noticed. The outer end of the drain (outside the abdominal cavity) was attached with monofilament suture. Then the inner end of the drain (in the anastomotic dehiscence) was grabbed with the endoscopic forceps and pulled together with an endoscope out through the mouth. A special device (a nasogastric tube embedded with sutured polyurethane sponge) was created and attached with the inner end of the drain pulled out through the mouth (Photo 2). Thus under the control of the endoscope and stabilizing gently with the monofilament suture outside the abdominal cavity, the nasogastric tube embedded with polyurethane sponge (E-VAC) was placed in the anastomotic leakage area (Photos 3, 4). The entire duration of the endoscopy did not exceed $20 \mathrm{~min}$. There were neither intraoperative nor postoperative complications. Immediately following placing of the E-VAC at the anastomotic dehiscence the NPWT was initiated. Continuous negative pressure of $100 \mathrm{~mm} \mathrm{Hg}$ was set up. At day 2 of treatment a significant decrease of secretions from one of the left outside drains was observed, whereas the total volume of exudate of E-VAC was approximately $1000 \mathrm{ml} /$ day. A gradual improvement of the patient's general con- 
dition was observed. According to the ultrasound scan there was no retention of any fluids within the abdominal cavity. To avoid ingrowing of the polyurethane sponge with granulated tissue, every day the E-VAC was slightly moved. At day 6 following initiated E-VAC therapy, a check-up with endoscopy was performed. The size of the anastomotic fistula significantly decreased, and there was no indication for further NPWT. Gradually the volume of discharge from the drain outside the abdominal cavity was decreasing. At day 14 following the NPWT the discharge was approximately $10-20 \mathrm{ml} /$ day. The patient was discharged from hospital at day 21 after the NPWT was implemented with no signs of pancreatico-gastric anastomotic dehiscence.

\section{Discussion}

The most common problem after surgical resection for esophageal carcinoma remains intrathoracic esophageal anastomotic leakage. The prevalence of cervical anastomotic leakage (mostly after esophagectomy) ranges from $10 \%$ to $25 \%$ [12]. Anastomotic leakage is a life-threatening complication associated

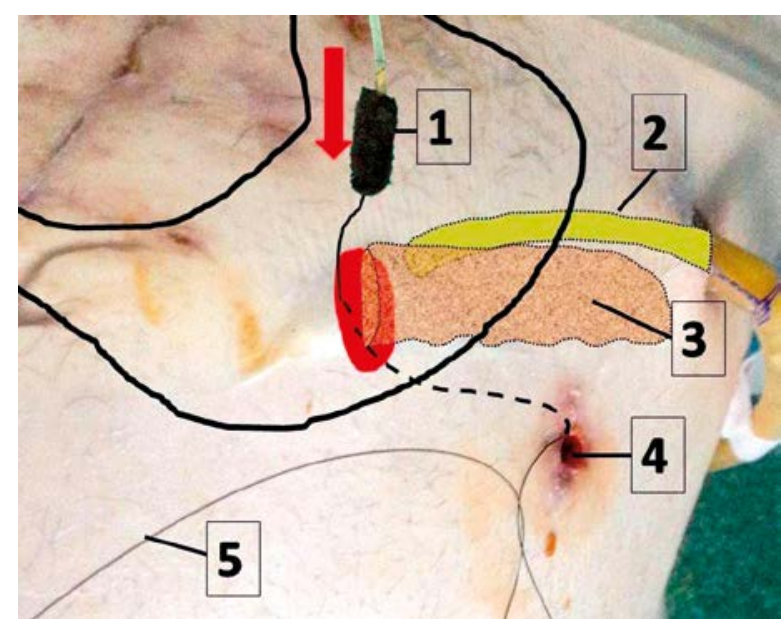

Photo 1. Details of endoscopic vacuum therapy in a patient after a leakage of the gastro-pancreatic anastomosis (leak highlighted in red, pancreas marked with [3]). The nasogastric tube with black sponge [1] is introduced to the stomach endoscopically, using suture [5], similar as in PEG technique. The suture has been previously attached to the drain, which was removed endoscopically through the stomach and esophagus. Abdominal wall opening after the drain is marked with [4], drain localized close to the anastomotic leakage is marked with [2] with a mortality rate between $3 \%$ and 10\% [12]. Treatment of this problem is challenging (particularly above the upper esophageal sphincter), and traditionally it is repaired by surgical intervention. However, this method is associated with high morbidity and mortality rates [13]. Clinically silent leakage and contained anastomotic leakage may be treated by conservative treatment (antibiotics and post-pyloric feeding).

The majority of leakages are currently treated by endoscopic methods such as self-expanding metal

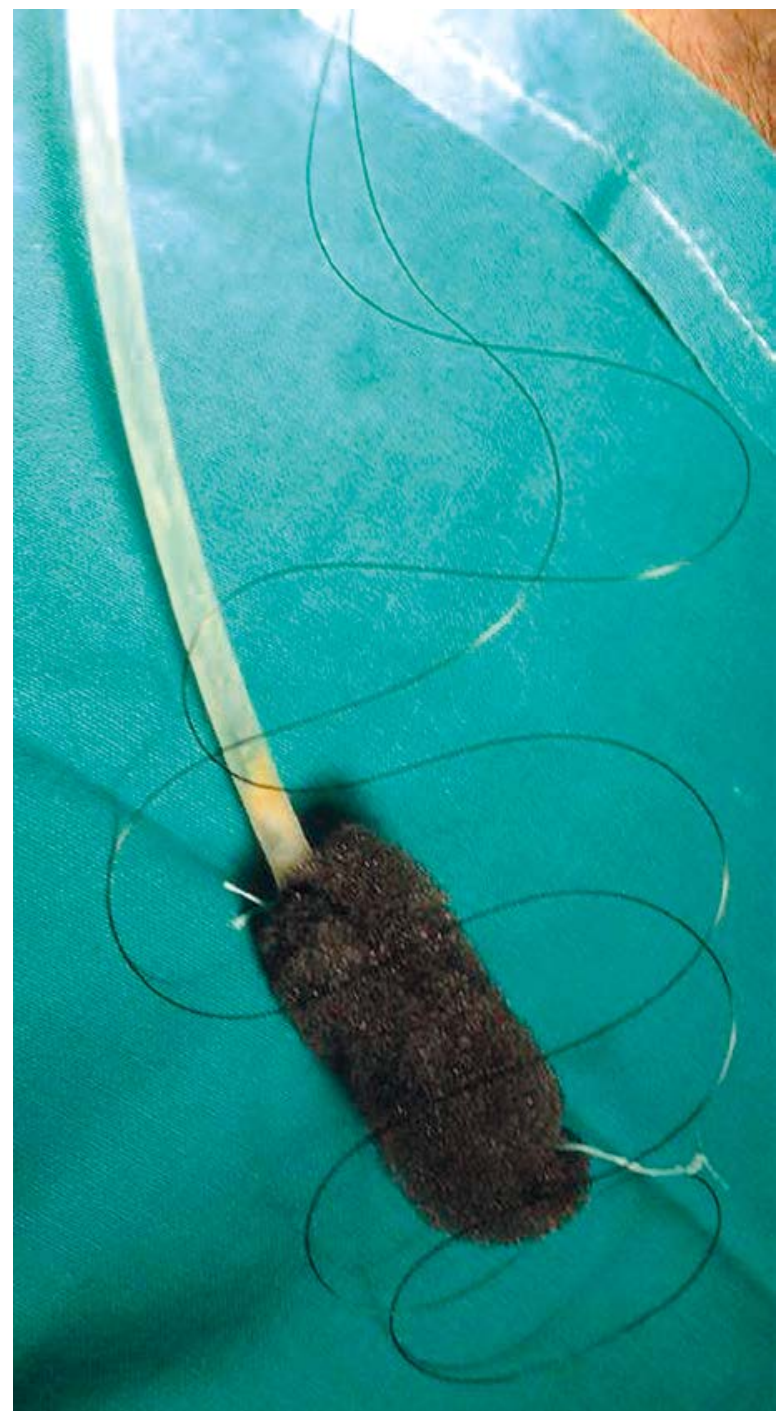

Photo 2. Nasogastric tube is connected with black sponge. The form and size of the sponge is similar to the size and form of the anastomotic leakage. Sponge is stabilized with suture to avoid the tube falling out and leakage being left in. Then the suture introduced by the place after the drain is stabilized to the sponge 


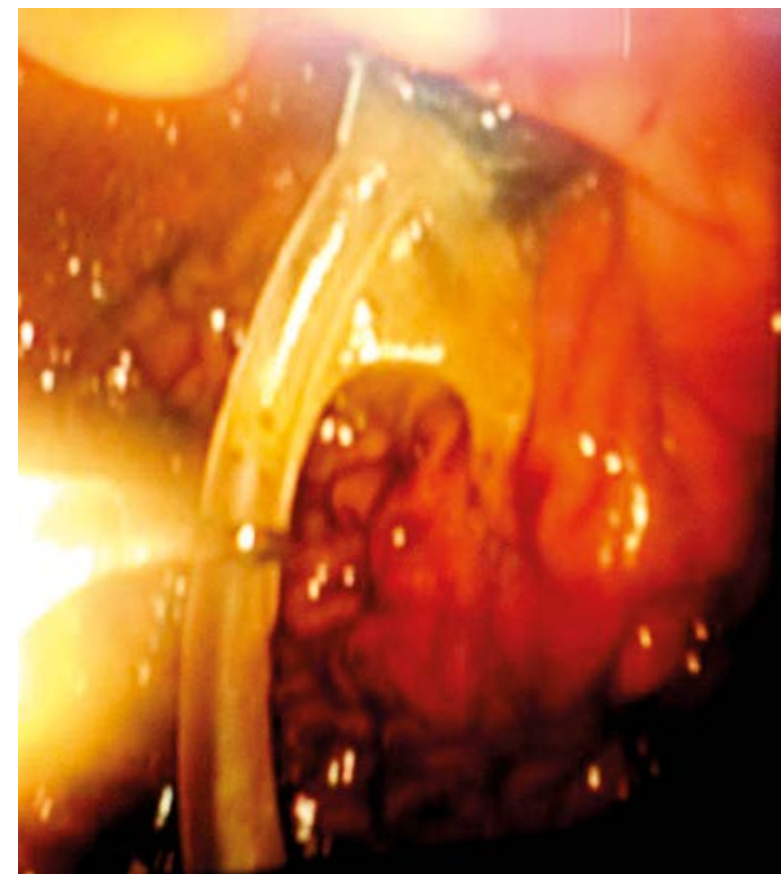

Photo 3. Endoscopic view of tube application carried through the stomach using forceps stents or plastic stents (SEMS/SEPS). These systems have become the treatment of choice for anastomotic esophageal leakage with success rates above 67-100\% [6]. Other methods include fibrin glue injection, endoscopic clips and vicryl plugs $[14,15]$.

Recently, E-VAC treatment has been suggested to be a potentially effective treatment option for upper gastrointestinal tract leakage. The first report about E-VAC was published in 2008 by Wedemeyer et al. They described 2 patients with anastomotic leakage after intrathoracic esophagogastrostomy and esophagojejunostomy due to esophagus carcinoma. In the first patient E-VAC therapy was applied secondary to failure of the SEMS procedure (SEMS was interrupted after 57 days) and in the second patient E-VAC was the primary and fully successful treatment [16].

The subsequent report by the same researchers described 8 patients treated with E-VAC. All patients had major postsurgical intrathoracic leakages, 6 after esophagojejunostomy, 1 after esophagogastrostomy and 1 after resection of diverticula. The authors used a specific and individually fashioned polyurethane foam/sponge. The sponge was intro-

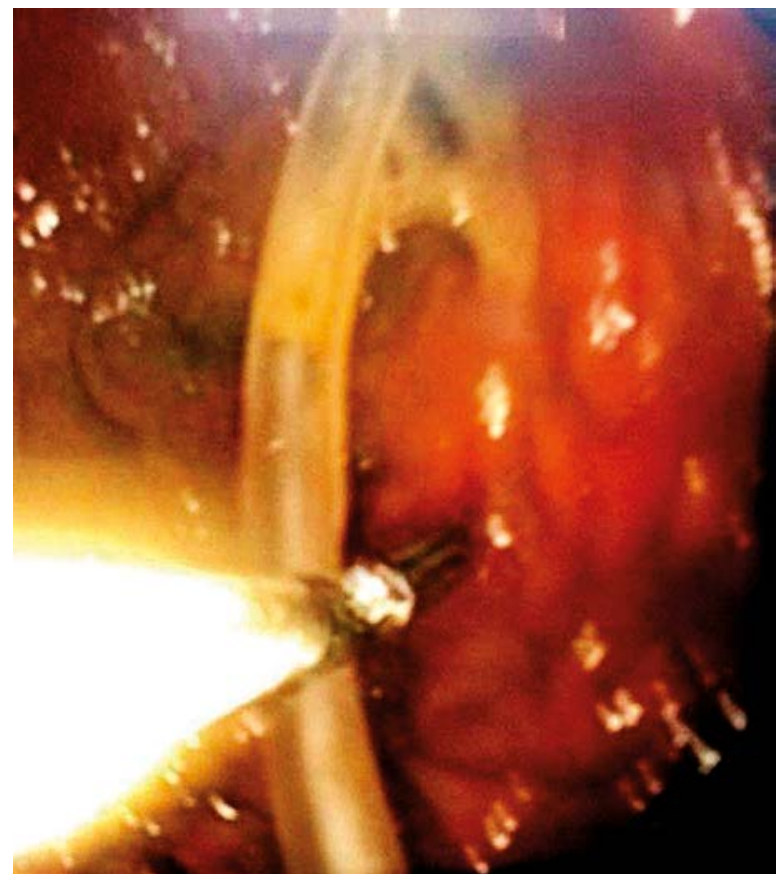

Photo 4. Endoscopic view of the last stage of the procedure - nasogastric tube with the sponge introduced into the leakage, good stabilization of the position possible by the suture derived through the abdominal wall

duced endoscopically in the necrotic cavity by a forward-viewing endoscope, and negative pressure of $125 \mathrm{~mm} \mathrm{Hg}$ was applied by a vacuum device. According to recommendations for standard VAC systems, the sponge was exchanged twice a week. Closure was achieved in $88 \%$ of patients (7 out of 8 ). A median of 7 endoscopic interventions were required, and the mean time of E-VAC treatment was 23.0 \pm 7.6 days [17].

Brangewitz et al. in a retrospective analysis compared 39 patients treated with SEMS/SEPS and 32 patients treated with E-VAC due to intrathoracic leakage. They concluded that successful wound closure was independently associated with E-VAC treatment (hazard ratio 2.997, 95\% confidence interval $(95 \% \mathrm{Cl}) 1.568-5.729 ; p=0.001)$. The closure rate was significantly higher in patients treated with E-VAC than SEMS/SEPS (84.4\% vs. 53.8\%). In addition, more strictures were found in the stent group in comparison to the E-VAC group (28.2\% vs. 9.4\%, $p<0.05)$. However, no difference was found in hospital mortality [18].

Ahrens et al. used an endoscopically guided endoluminal vacuum therapy in 5 patients with major 
leakage from an esophageal anastomosis. Polyurethane sponges were endoscopically positioned in the wound cavity of the anastomosis. Continuous negative pressure was applied via drainage tubes with polyurethane sponge and endoscopically changed three times per week. In all patients treatment was successful. The wound cavity size ranged from $42 \mathrm{~cm}$ to $157 \mathrm{~cm}$. The median time of drainage was 28 days (median time to total cavity closure was 42 days), with a median of 9 sponge changes. One patient died because of an acute severe hemorrhage from aorto-anastomotic fistula [19].

Weidenhagen et al. also evaluated the applicability of E-VAC after esophagectomy. Their study included 6 patients with intrathoracic anastomotic leakage after esophageal resection. All patients were treated with mediastinal E-VAC without any local complications or the need for reoperation [5].

Kuehn et al. reported a series of 9 patients with E-VAC therapy. The indication for E-VAC therapy was anastomotic leakage after esophageal resection or gastrectomy (5 patients) and iatrogenic or spontaneous esophageal perforations (4 patients). The mean number of sponge insertions was 6 (range: 1-13) with a mean changing interval of 3.5 days (range: 2-5). Successful E-VAC therapy was achieved in $89 \%$ (8 out of 9 patients). In 4 patients, initial E-VAC therapy was performed in combination with open surgical revision [20].

Loske et al. described 10 patients treated with E-VAC therapy due to anastomotic insufficiency secondary to esophagectomy or gastrectomy (5 patients), iatrogenic esophageal perforation (2 patients), esophageal wall necrosis (1 patient), Boerhaave's syndrome (1 patient), and perforation of esophageal cancer (1 patient). The mean time of treatment was 12 days, with a mean number of sponge placements of 3 and mean replacement interval of 2-7 days. Defects were healed in all the surviving patients. One of the patients died because of fulminant pseudomembranous colitis. During follow-up (10-380 days after E-VAC therapy) the authors did not find any stenosis or functionally relevant scar formation [21].

Loske et al. also published a case report about application of intracavitary E-VAC therapy in Boerhaave's syndrome. A 78-year-old patient was diagnosed with Boerhaave's syndrome after gastroscopy. A procedure revealed a $2 \mathrm{~cm}$ esophageal defect that opened into an abscess cavity with $5 \mathrm{~cm}$ diameter.
A polyurethane foam sponge was inserted and secured by suture. The vacuum device was connected (with negative pressure of $125 \mathrm{~mm} \mathrm{Hg}$ ) and the sponge was changed once on the $4^{\text {th }}$ day. After 8 days of E-VAC therapy the size of the cavity was significantly reduced and E-VAC therapy was stopped. In endoscopic follow-up after 10 days, a small scar without any stenosis was visible and the patient had no difficulty with swallowing [22].

The utility of E-VAC in management of anastomotic leakages was also assessed by Schniewind et al. The study group consisted of 62 patients with esophageal leakage out of 366 who underwent esophagectomy. Therapy regimens included surgical revision (18 patients), E-VAC therapy (17 patients), SEMS/SEPS application (12 patients), and conservative management (15 patients). The E-VAC therapy was used among 17 (27.4\%) patients. Continuous negative pressure of $70-80 \mathrm{~mm} \mathrm{Hg}$ was applied, with a median of 3 sponge changes per week. The E-VAC therapy was ended when the wound cavity diameter was $2 \mathrm{~cm}$ or less. The authors analyzed various types of data such as scores on the APACHE II, in-hospital mortality, cervical leakage and mean in-hospital time and time in the ICU. However, compared groups of patients were not homogeneous and significantly differed from the conservative group. The APACHE II score was $5 \pm 2$ in the conservative versus $14 \pm 4$ in the E-VAC group, $15 \pm 5$ in the surgical treatment group and $11 \pm 3$ in the stent treatment group. Inhospital mortality was significantly lower in the E-VAC group (12\%; 2 patients) in comparison to the surgical treatment group (50\%; 9 patients) and stent treatment group (42\%; 5 patients). The survival rate in the E-VAC group was significantly superior to the surgically and stent treated patients ( $p=0.011$ and $p=0.00014)$. The authors postulated that E-VAC therapy may be an effective procedure for the management of major leakage from esophageal anastomoses and may be better than surgical and stent intervention [23].

Lenzen et al. described 3 male patients with post-operative cervical esophageal leakage treated with E-VAC therapy. The primary cause of the leakage in 1 patient was the state after surgical excision of a pharyngo-cervical liposarcoma with partial esophageal resection, and the other 2 patients underwent surgical treatment of Zenker's diverticulum. The endo-sponge was endoscopically positioned into the wound cavity, and when the cavity wound 
size was decreased, the sponge was positioned intraluminally to cover the leak. Continuous negative pressure of $125 \mathrm{~mm} \mathrm{Hg}$ was applied, and the VAC system was changed twice a week. The median duration of the E-VAC therapy drainage was 29 days (range: 19-49 days), with a median of 7 sponge exchanges (range: 5-12 exchanges). The E-VAC therapy was successful for all patients; complete closure of the esophageal leak was achieved without any procedure-related complications (without problems with swallowing and no evidence of stricture). Furthermore, the authors reported that E-VAC therapy reduced inflammatory markers [24].

Gubler et al. reported 2 patients with distal esophagus carcinoma treated by esophagectomy and intrathoracic anastomosis. Both cases developed an anastomosis insufficiency. The primary approach in the first case consisted of insertion of an endo-sponge into the wound cavity and a suction tube was connected to a continuous negative pressure (75-100 mm Hg). Additionally, the endo-sponge was overstented by SEMS (15 cm length and $20 \mathrm{~mm}$ diameter). In the second case, E-VAC therapy was a procedure secondary to failure of insertion of a partially covered self-expanding metallic stent. The endo-sponge was also covered by a stent $(15 \mathrm{~cm}$ length). Finally, in both patients the leakages were closed respectively 26 days and 12 weeks after esophagectomy [25].

Schorsch et al. evaluated the utility of E-VAC therapy (intraluminal or intracavitary) in 17 patients with anastomotic leakage and in 7 patients with iatrogenic perforation (due to interventional endoscopy or rigid panendoscopy). Patients with iatrogenic perforation were treated mostly by the intraluminal method (1 case required both techniques), and patients with anastomotic leakage were treated mostly by intracavitary E-VAC therapy (3 patients required application of intraluminal and intracavitary techniques). The E-VAC treatment was successful for $96 \%$ of patients (23 out of 24 cases; only 1 patient with anastomotic leakage was not cured). The median duration of therapy was 11 days (range: 4-46 days). In all patients continuous negative pressure of $125 \mathrm{~mm} \mathrm{Hg}$ was applied. Follow-up demonstrated one case of esophageal stenosis after circular anastomotic insufficiency (managed by endoscopic balloon dilation) [26].

In the next analysis, Schorsch et al. described 35 patients treated with E-VAC therapy (21 had anas- tomotic leakage, 7 had iatrogenic perforation and 7 patients had esophageal defects of various other origins). Intraluminal or intracavitary drainage systems with an open pore polyurethane tip were applied using a standard endoscope. The E-VAC was connected to a standard vacuum device and continuous negative pressure of $125 \mathrm{~mm} \mathrm{Hg}$ was maintained. In 91.4\% (32 out of 35 patients) healing of defects was achieved with a median treatment duration of 11 days (range: 4-78 days). The postoperative anastomotic leakage healed in $95.2 \%$ (20 out of 21 patients) with a median of 11 days (range: 4-46 days). All patients with iatrogenic perforation defects were healed after a median treatment time of 5 days (range: 4-7 days). The authors did not record a case of recurrent fistula after 75 days of treatment. The 90-day mortality was $5.7 \%$ [27].

Bludau et al. reported 14 patients with esophageal defects treated with E-VAC (3 with spontaneous defect, 2 with iatrogenic defect, and 9 with postoperative esophageal defect). The average duration of E-VAC application was 12.1 days with an average of 3.9 E-VAC systems used. For $42.85 \%$ (6 out of 14 patients), the authors used combined therapy with E-VAC and the placement of SEMS. The reinstatement of the esophageal defect was achieved in $86 \%$ (12 of the 14 patients). The authors reported 2 deaths due to severe sepsis [28].

Heits et al. assessed the value of the endo-sponge in 10 patients with iatrogenic, spontaneous, or foreign body-associated perforation. The mean number of endo-sponges installed was 5.4 (range: 2-12) with a mean period of treatment of $19 \pm 14.26$ days. Successful E-VAC therapy was achieved in $90 \%$ (9 out of 10 patients). After primary treatment, 1 patient had additional SEMS insertion and 1 patient needed surgical resection due to repeated Mallory-Weiss lesions and perforations after immunosuppressive therapy. One patient died due to general failure of the cardiovascular system [29].

The other problem is anastomotic leakages after rectal surgery. They are major complications (incidence ranges from $3 \%$ to $19 \%$ ) after rectal surgery and the main cause of postoperative morbidity and mortality [9, 30]. Preoperative factors that predispose to anastomotic leakage are still not fully known. So far there is no treatment of choice for management of rectal anastomotic leakage. Available methods of treatment include a wide range of surgical procedures such as drainage, loop colostomy, resection 
of the anastomosis with colostomy, Hartmann procedure or abdominoperitoneal extirpation and conservative treatments (nasogastric suction, antibiotic coverage and parenteral nutrition) [31].

The best choice from various procedures depends on the level of the anastomotic leakage, the patients' clinical state, the efficiency of the secretion drainage, the time point and many other factors. Generally, conservative treatment can be routine therapy for the patient without generalized peritonitis, but the process of healing is often slower and the functional outcome can be unsatisfactory.

In some departments and clinics, transanal E-VAC therapy, endoscopic transanal vacuum-assisted rectal drainage (ETVARD) or Endo-SPONGE therapy has been established as the routine treatment for patients with major rectal anastomotic leakage without sepsis. The E-VAC treatment is also useful among patients with leakage after neoadjuvant chemoradiotherapy, iatrogenic perforations and anastomotic fistulas [4, 32-34].

The first report on transanal E-VAC application was published by Wiedenhagen et al. in 2003 in the form of an abstract without any specifications of the technique. The authors described a new method for control of sepsis caused by anastomotic leakage in rectal surgery - the transanal E-VAC.

Nagell et al. described 4 patients with anastomotic leakage after rectal resections treated with E-VAC, and 10 patients treated conservatively in the previous 5 years as the control group. Median healing time for E-VAC patients was 51 days (43-195 days), and for the control group it was 336 days (52-1,464 days). No side effects during or after the E-VAC were observed, and the therapy was perfectly tolerated by all patients [33].

Richterich et al. reported a new application for the Endo-SPONGE. The authors used the E-VAC system for a patient with iatrogenic perforation after diagnostic colonoscopy. Duration of E-VAC therapy was 9 days, and through this time the endo-sponge was installed only once. The final result was excellent, and the patient tolerated therapy without any complications or side affects [35].

Weidenhagen et al. assessed the utility of transanal E-VAC in 29 patients with anastomotic leakage after anterior rectal resection. Total duration of E-VAC therapy was $34.4 \pm 19.4$ days with an average number of endoscopic procedures of $11.4 \pm 6.3$. Therapy was ended when the depth of the cavity was less than $0.5 \times 1 \mathrm{~cm}$. The mean postoperative hospital stay was $30.5 \pm 12.8$, and in 25 patients therapy was continued ambulatory. A significant decrease of C-reactive protein concentration (CRP) was observed after 7 days of treatment (from $14.0 \mathrm{mg} / \mathrm{dl}$ to $2.9 \mathrm{mg}$ / $\mathrm{dl}, p<0.05)$. Complete healing was achieved in nearly all the study group (28 out of 29 patients). A follow-up study revealed a therapy-relevant stenosis in the anastomotic region in 10 patients. Dilatation procedures were necessary in $5.8 \pm 2.2$ patients. In 21 of the 29 patients, a protecting stoma was created during the primary procedure. Five patients were treated primarily by E-VAC therapy (4 of them without secondary stoma), and 1 of them needed a secondary stoma to control the infection. Another 3 patients required primarily an immediately operation due to generalized peritonitis [4].

Mees et al. reported a series of 10 patients with anastomotic leakage after colorectal resections. $\mathrm{Pa}$ tients were treated with an E-VAC (5 patients) or by transrectal lavage (5 patients). Median duration of E-VAC therapy was 27 days. Time of wound healing was significantly accelerated in the E-VAC group as compared to the group treated with transrectal lavage. In-hospital time was shortened in the E-VAC group. However, assessment of the pain in both groups did not reveal significant differences [36].

Glitsch et al. described a group of 17 patients with anastomotic leakage after resection of the rectum or rectosigmoid colon. An endo-sponge was installed into the wound cavity via endoscopic techniques and continuous negative pressure was applied. In nearly $100 \%$ (16 out of 17 ) of patients transanal E-VAC therapy was successful. Only 1 patient eventually required a Hartmann's procedure. Additionally, 15 patients received intramural fibrin glue injections. The mean duration of E-VAC therapy was 21.4 days, mean number of sponge changes was 5.4, and mean number of endoscopies was 10.7. Mean time to closure of the wound cavity was 53.1 days. The total time to closure was dependent on the primary size of the cavity $(p<0.015)$. The healing time also depended significantly on age of the patients $(p<0.036)$. When anastomoses were located $6 \mathrm{~cm}$ or less from the anocutaneous line, patients had considerably longer healing times. Endoscopy follow-up showed minor anastomotic leakage in 2 patients [37].

Van Koperen et al. described 2 patients after restorative proctocolectomy for ulcerative colitis with 
J-pouch reconstruction. Anastomotic leakage without general peritonitis developed in both cases. The endoscopic transanal placement of an endo-sponge was performed secondarily to diverting ileostomy. The endo-sponge was connected to a low suction bottle (Redyrob Trans Plus suction device), and the sponge was changed every 3-4 days. Final and acceptable results were achieved after 35 and 56 days [38].

Von Bernstorff et al. evaluated 26 patients with anastomotic leaks after rectal resection due to a malignant process. Fourteen patients followed neoadjuvant radiochemotherapy before surgery. The E-VAC therapy was the first-line treatment in all patients, and in 23 patients was successfully completed. Patients following neoadjuvant radiochemotherapy had an increased time to leakage cavity closure. Moreover, they had bigger sizes of leakage cavities, longer duration of E-VAC therapy, and more endoscopies and sponge exchanges $(0.009<p<0.035)$ as compared to patients without neoadjuvant radiochemotherapy. Similar results as patients who underwent neoadjuvant radiochemotherapy were found among patients older than 62 years. Older age influenced the time to closure of the cavity ( $p$ < 0.029). However, other correlations were not statistically significant. The authors claimed that patients without (ile)ostomies could also be treated by E-VAC therapy with good results. The follow-up study did not reveal any complications [32].

Van Koperen et al. in a multicenter Dutch experience described a series of 16 patients after surgery due to rectal cancer (13 patients) or ulcerative colitis (3 patients) treated with E-VAC therapy because of anastomotic leakage. Fifty percent of patients started treatment with the endo-sponge within 6 weeks after the initial surgery (after a median of 24 days, range: $13-39$ days). The remaining $50 \%$ of patients started E-VAC therapy 6 weeks or later after primary surgery (after a median of 74 days, range: 43-1,602 days). The closure was successful in $75 \%$ of patients ( 6 out of 8 ) in the first group compared to $38 \%$ of patients (3 out of 8 ) in the second group ( $p=0.315)$. The total rate of cavity closure was $56 \%$ ( 9 cases). The mean anastomotic level was $5 \mathrm{~cm}$ from the anal verge (range: $2-8 \mathrm{~cm}$ ). In the whole group closure was achieved after a median of 40 days (range: 28-90) with a median of 13 sponge replacements (range: $8-17)$. The authors created 8 protective stomas during the primary surgery, and 9 patients had preoperative radiotherapy due to rectal cancer [39].
In 2010 Riss et al. reported 9 patients with anastomotic leakage after anterior rectal resection (6 patients) or leakage of the rectal stump after Hartmann's procedure (3 patients). All leakages were treated with the E-VAC procedure with continuous negative pressure of $100 \mathrm{~mm} \mathrm{Hg}$. One patient received short neoadjuvant radiotherapy, 3 patients underwent neoadjuvant chemoradiotherapy, and 1 had at the moment of diagnosis metastases in the liver. Two patients received chemoradiotherapy after the primary operation. Pain sensation during E-VAC therapy was assessed using a 10-point visual analogue scale (VAS). Moreover, quality of life was evaluated by the author's specific questionnaire. Median time of E-VAC treatment was 21 days (range: 2-8). There were no minor or major complications. In $66.6 \%$ of patients, the anastomotic leakage healed completely. The failure of E-VAC was due to complexity of the leakage or was affected by chemotherapy. Six patients declared their willingness for re-treatment with E-VAC if necessary. The median VAS score was 3 (range: 0-6) [40].

A subsequent study by the same authors enrolled 20 patients with primary successful E-VAC treatment of anastomotic leakage after rectal cancer resection. The E-VAC treatment was applied in 17 patients with anastomotic leakage and in 3 patients with inefficiency of the rectal stump after Hartmann's procedure. Patients were divided into 2 groups: with a successful outcome (75\%) and without a successful outcome (25\%). The median duration of E-VAC was 21 days in both groups. Patients without a successful outcome developed a recurrent symptomatic abscess after E-VAC. Median time between ending of E-VAC therapy and appearance of recurrent abscess was 255 days (range: 21733 days). Median interval between operation and occurrence of anastomotic leakage was statistically longer $(p<0.05)$ in the group without a successful long-term outcome. Two patients (10\%) developed a recurrence of local tumor and afterwards died. The median follow-up duration was 16.1 months in the first group and 17 months in the second group [41].

Hoogenboom et al. described a 70-year-old man with small intestinal-colorectal anastomotic fistula developing during endo-sponge treatment. The patient was diagnosed with $\mathrm{T} 3 \mathrm{~b}$ rectal carcinoma and underwent preoperative chemoradiotherapy and laparoscopic low anterior rectum resection. Postoperatively, an anastomotic leakage was investigated by 
CT. Three weeks after the operation E-VAC was initiated, with the sponge being changed every 3-4 days. Initially, the abscess cavity granulated, but after 10 weeks of therapy, an enterocutaneous small intestinal fistula developed. The patient was treated surgically 4 weeks later without any further complications [42].

Einenkel et al. described a 44-year-old woman who underwent complete ovarian cancer removal (FIGO IIIC) with final colorectal end-to-end anastomoses (by circular stapling). Transvaginal ultrasound performed 12 days after the operation revealed a colorectal anastomotic dehiscence. The leakage was successfully managed by transanal E-VAC. The patient was discharged on postoperative day 29 with complete recovery [43].

Arezzo et al. presented 3 patients treated with E-VAC therapy due to rectal anastomotic leakage and anastomotic fistula after different surgical procedures (1 case after an internal Delorme procedure and the 2 others after total mesorectal excision with diverting loop ileostomy). In all cases endoscopic procedures revealed an abscess cavity, and intracavitary E-VAC (Endo-SPONGE) therapy was introduced. In the first case the abscess cavity had a connection with the vagina and the Endo-SPONGE was replaced every $48 \mathrm{~h}$. In the second case the patient underwent neoadjuvant chemoradiotherapy, and 3 months after primary surgery presacral local recurrence was diagnosed. Colonoscopy showed a small anastomotic fistula, and after balloon dilatation a larger presacral abscess cavity was entered. An Endo-SPONGE was installed intracavitarily, and after 2 months of therapy the cavity size decreased to $12 \times 10 \mathrm{~mm}$. The loop ileostomy was closed after 30 months. In the third case the patient was diagnosed 4 weeks after primary surgery with a circumferential leakage and large abscess cavity without any communication with the peritoneal cavity. An Endo-SPONGE was placed and changed twice after $48 \mathrm{~h}$ and $96 \mathrm{~h}$. After $96 \mathrm{~h}$ of treatment the CT scan showed a large amount of air, and emergency proctectomy with end colostomy was performed [34].

Recently, Rosenberger et al. published a paper about application of endoluminal VAC in Yorkshire pigs who underwent rectal resection. The authors confirmed an intentional leak by fluoroscopy, and an endoluminal VAC device was placed. Necropsy was performed for histopathology. The pigs were divided into two subgroups according to the duration of free intraperitoneal leakage: early-treated (2 days of free leakage) and late-treated (5 days of free leakage). The early-treated subgroup underwent 5-day E-VAC therapy, but the late-treated subgroup underwent 7-day E-VAC therapy. In 6 out of 7 early-treated pigs the anastomotic defect sealed after E-VAC application. However, the defect closed in only 2 of 4 late-treated pigs. The authors concluded that E-VAC therapy is practicable and well tolerated in a pig model and may be a new noninvasive technique for treatment of anastomotic leakages. However, better results were observed in the early period (86\% completely healed anastomotic leakage) [44].

Wedemeyer et al. was the first researcher to apply transgastrically placed E-VAC therapy as an addition to transgastric necrosectomy in necrotizing pancreatitis. A 56-year-old male patient was on immunosuppressive therapy after kidney transplantation. After many days of unsuccessful endoscopic treatment and intensive antibiotic therapy, E-VAC therapy was introduced. E-VAC therapy was performed for 20 days and the sponge was changed 5 times. The authors noted one bleeding episode following the sponge exchange procedure without severe complications. After ending E-VAC therapy, no pancreatitis-associated complications were observed. The second patient was a 52-year-old man with metastatic colon cancer and necrotizing pancreatitis with a perisplenic abscess. The abscess developed 21 days after the onset of symptoms. The patient underwent complex surgical and endoscopic treatment with E-VAC therapy. The endo-sponge was exchange twice and was removed after 6 days of treatment [45].

D'Hondt et al. reported a case of a 76-year-old woman after anterior resection of the rectum for an adenocarcinoma. Eight years later the patient underwent a complete proctectomy because of chronic proctitis with anal mucus secretion and blood loss. The CT scan revealed a large (about $11 \mathrm{~cm}$ ) presacral cavity. Biopsies with histologic examinations were negative for recurrence of the neoplastic process. An Endo-SPONGE was installed into the presacral cavity, and foam was exchanged every 4 days (initially under sedation and later continued as ambulatory treatment). The E-VAC was continued for 20 days when the presacral cavity was smaller than the size of foam. Five-month follow-up did not reveal any complications [46].

The first report about the usefulness of E-VAC therapy in the treatment of an infected pancreatic 
pseudocyst (IPC) was published by Wallstabe et al. A 28-year-old woman with severe inflammatory response syndrome (SIRS) because of IPC underwent transgastric puncture and endoprosthesis drainage (by double pigtail endoprosthesis). After 7 days the SIRS had resolved, but the pseudocyst still was not completely closed. The E-VAC therapy was introduced with negative pressure of $120 \mathrm{~mm} \mathrm{Hg}$. E-VAC therapy was completed on day 7. Eight-week follow-up did not reveal any complications [47].

Wallstabe et al. reported using a E-VAC for a patient who developed sepsis due to an infected pancreatic pseudocyst (IPC) and chronic pancreatitis. The authors removed the necrosis from the cyst by endoscopic therapy and after that started E-VAC with continuous negative pressure of $120 \mathrm{~mm} \mathrm{Hg}$. The authors used Suprasorb Drainage Film (Lohmann \& Rauscher, Vienna, Austria) wrapped around the Endo-SPONGE. They claimed that wrapped Endo-SPONGE extraction was easier as compared to pure sponge. Also transgastric access was smoother and less bloody. The pseudocyst closed after 7 days of treatment [48].

In 2013 Seyfried et al. described successful management of gastric leakage after Roux-en-Y gastric bypass (RYGB). The authors had twice previously used covered metal stents to seal the leakage without success (24 mm $\times 10 \mathrm{~cm}$; Beta-stent $26 / 34 \mathrm{~mm}$ $\times 12 \mathrm{~cm}$; both Taewoong Medical). In both cases the stents had migrated distally. Intracavitary E-VAC was installed into the wound cavity with continuous negative pressure of $125 \mathrm{~mm} \mathrm{Hg}$. The endo-sponge was changed every 3 days. Complete closure of the leakage was achieved after 1 week of E-VAC therapy [49].

Schorsch et al. reported a 65-year-old patient after a Kausch-Whipple procedure with gastropancreatic anastomosis. Postoperatively, endoscopy showed a semicircular dehiscence of the pancreaticogastric anastomosis. An intraluminal E-VAC was placed over the anastomosis with continuous negative pressure of $125 \mathrm{~mm} \mathrm{Hg}$. On day 8 there were no endoscopic signs of anastomotic leakage and E-VAC therapy was ended with a satisfactory result [50].

Fischer et al. published a case of a 73-year-old patient with pylorus-preserving pancreatic head resection because of branch-duct type intraductal papillary mucinous carcinoma. At day 14 after the procedure the CT scan confirmed pancreaticogastrostomy leakage. An Endo-SPONGE was introduced via a gastric tube into the abscess cavity, and
E-VAC therapy was applied with negative pressure of $30 \mathrm{~mm} \mathrm{Hg}$. The endo-sponge was changed twice. At day 8 after beginning E-VAC therapy a fully clean cavity was observed and therapy was terminated. At the same time, a rubber drain was introduced into the cavity and drawn back slowly over 17 days. The anastomotic leak and abscess cavity were healed completely after 26 days of therapy [51].

\section{Conclusions}

The E-VAC provides perfect wound drainage and closure of various kinds of defects and promotes tissue granulation. This therapy may significantly improve morbidity and mortality. Moreover, E-VAC may be useful in the multidisciplinary approach - from upper gastrointestinal to rectal surgery complications.

The E-VAC therapy may be an effective procedure for the management of major leakage from esophageal anastomoses $[23,26]$. Also E-VAC treatment might be superior to a surgical procedure and stent placement [23]. Likewise, E-VAC may be a routine choice for iatrogenic esophageal perforations [26]. The E-VAC is a very promising method for complex uncontained leaks to the chest cavity [25]. Recently, combined E-VAC and SEMS/SEPS therapy was also introduced [28].

Transanal E-VAC (or ETVARD) may be a first line treatment for patients with major leaks after rectal anastomoses. This therapy is effective in patients following neoadjuvant chemoradiotherapy and in patients with additional chemoradiotherapy [32]. However, other authors claimed that the effectiveness of E-VAC therapy is affected by chemoradiotherapy [40]. Most researchers suggested that the early E-VAC approach is probably better than the choice of late treatment [38]. Usually, it is a treatment well accepted by patients [40].

The E-VAC can also be a cost-effective procedure. The E-VAC avoids complicated, time- and cost-consuming open surgical reoperations. Also the total cost of treatment, by reducing the time of total parenteral nutrition, systemic antibiotics and intensive care, can be reduced in endoscopically treated patients.

On the other hand, major limitations of the E-VAC system are the necessity of repeated endoscopic interventions and constant presence of well-trained staff. Some authors suggested that the E-VAC method may be ineffective in the course of sepsis [28]. Furthermore, continuous negative pressure needs to 
be applied, and therefore patients require hospitalization. However, some clinicians have reported that E-VAC therapy may be managed in the outpatient clinic. Only one paper reported a $25 \%$ rate of unsuccessful long-term outcome after the transanal E-VAC procedure [41].

Further extensive, large-cohort studies, also including an economic analysis, need to be performed to establish the applicability and effectiveness of E-VAC, before routine widespread use can be recommended.

\section{Conflict of interest}

The authors declare no conflict of interest.

\section{References}

1. Krokowicz L, Borejsza-Wysocki M, Mackiewicz J, et al. 10 years of negative pressure wound therapy [NPWT]: evolution of indications for its use. Negative Pressure Wound Therapy 2014; 1: 27-32.

2. Argenta LC, Morykwas MJ. Vacuum-assisted closure: a new method for wound control and treatment: clinical experience. Ann Plast Surg 1997; 38: 563-576; discussion 577.

3. Banasiewicz T, Borejsza-Wysocki M, Meissner W, et al. Vacuum-assisted closure therapy in patients with large postoperative wounds complicated by multiple fistulas. Videosurgery Miniinv 2011; 6: 155-63.

4. Weidenhagen R, Gruetzner KU, Wiecken T, et al. Endoscopic vacuum-assisted closure of anastomotic leakage following anterior resection of the rectum: a new method. Surg Endosc 2008; 22: 1818-25.

5. Weidenhagen R, Hartl WH, Gruetzner KU, et al. Anastomotic leakage after esophageal resection: new treatment options by endoluminal vacuum therapy. Ann Thorac Surg 2010; 90: 1674-81.

6. Safranek J, Geiger J, Vesely V, et al. Esophageal stents for less invasive treatment of mediastinitis. Videosurgery Miniinv 2014; 9: 1-5.

7. Wu PC, Posner MC. The role of surgery in the management of oesophageal cancer. Lancet Oncol 2003; 4: 481-8.

8. Kruschewski M, Rieger H, Pohlen U, et al. Risk factors for clinical anastomotic leakage and postoperative mortality in elective surgery for rectal cancer. Int J Colorectal Dis 2007; 22: 919-27.

9. Lee WS, Yun SH, Roh YN, et al. Risk factors and clinical outcome for anastomotic leakage after total mesorectal excision for rectal cancer. World I Surg 2008; 32: 1124-9.

10. Jung SH, Yu CS, Choi PW, et al. Risk factors and oncologic impact of anastomotic leakage after rectal cancer surgery. Dis Colon Rectum 2008; 51: 902-8.

11. Buchs NC, Gervaz P, Secic M, et al. Incidence, consequences, and risk factors for anastomotic dehiscence after colorectal surgery: a prospective monocentric study. Int J Colorectal Dis 2008; 23: 265-70.

12. Larburu Etxaniz S, Gonzales Reyna J, Elorza Orue JL, et al. Cervical anastomotic leak after esophagectomy: diagnosis and management. Cir Esp 2013; 91: 31-7.
13. Jougon J, Delcambre F, MacBride T, et al. Mortality from iatrogenic esophageal perforations is high: experience of 54 treated cases. Ann Chir 2002; 127: 26-31.

14. Bohm G, Mossdorf A, Klink C, et al. Treatment algorithm for postoperative upper gastrointestinal fistulas and leaks using combined vicryl plug and fibrin glue. Endoscopy 2010; 42: 599-602.

15. Raju GS. Endoscopic clip closure of gastrointestinal perforations, fistulae, and leaks. Dig Endosc 2014; 26 Suppl 1: 95-104.

16. Wedemeyer J, Schneider A, Manns MP, Jackobs S. Endoscopic vacuum-assisted closure of upper intestinal anastomotic leaks. Gastrointest Endosc 2008; 67: 708-11.

17. Wedemeyer J, Brangewitz M, Kubicka S, et al. Management of major postsurgical gastroesophageal intrathoracic leaks with an endoscopic vacuum-assisted closure system. Gastrointest Endosc 2010; 71: 382-6.

18. Brangewitz M, Voigtlander T, Helfritz FA, et al. Endoscopic closure of esophageal intrathoracic leaks: stent versus endoscopic vacuum-assisted closure, a retrospective analysis. Endoscopy 2013; 45: 433-8.

19. Ahrens M, Schulte T, Egberts J, et al. Drainage of esophageal leakage using endoscopic vacuum therapy: a prospective pilot study. Endoscopy 2010; 42: 693-8.

20. Kuehn F, Schiffmann L, Rau BM, Klar E. Surgical endoscopic vacuum therapy for anastomotic leakage and perforation of the upper gastrointestinal tract. J Gastrointest Surg 2012; 16: 2145-50.

21. Loske G, Schorsch T, Muller C. Endoscopic vacuum sponge therapy for esophageal defects. Surg Endosc 2010; 24: 2531-5.

22. Loske G, Schorsch T, Muller C. Endoscopic intracavitary vacuum therapy of Boerhaave's syndrome: a case report. Endoscopy 2010; 42 Suppl 2: E144-5.

23. Schniewind B, Schafmayer C, Voehrs G, et al. Endoscopic endoluminal vacuum therapy is superior to other regimens in managing anastomotic leakage after esophagectomy: a comparative retrospective study. Surg Endosc 2013; 27: 3883-90.

24. Lenzen H, Negm AA, Erichsen TJ, et al. Successful treatment of cervical esophageal leakage by endoscopic-vacuum assisted closure therapy. World J Gastrointest Endosc 2013; 5: 340-5.

25. Gubler C, Schneider PM, Bauerfeind P. Complex anastomotic leaks following esophageal resections: the new stent over sponge (SOS) approach. Dis Esophagus 2013; 26: 598-602.

26. Schorsch T, Muller C, Loske G. Endoscopic vacuum therapy of anastomotic leakage and iatrogenic perforation in the esophagus. Surg Endosc 2013; 27: 2040-5.

27. Schorsch T, Muller C, Loske G. Endoscopic vacuum therapy of perforations and anastomotic insufficiency of the esophagus. Chirurg 2014; 85: 1081-93.

28. Bludau M, Holscher AH, Herbold T, et al. Management of upper intestinal leaks using an endoscopic vacuum-assisted closure system (E-VAC). Surg Endosc 2014; 28: 896-901.

29. Heits N, Stapel L, Reichert B, et al. Endoscopic endoluminal vacuum therapy in esophageal perforation. Ann Thorac Surg 2014; 97: 1029-35.

30. Branagan G, Finnis D. Prognosis after anastomotic leakage in colorectal surgery. Dis Colon Rectum 2005; 48: 1021-6.

31. Mileski WJ, Joehl RJ, Rege RV, Nahrwold DL. Treatment of anastomotic leakage following low anterior colon resection. Arch Surg 1988; 123: 968-71. 
32. von Bernstorff W, Glitsch A, Schreiber A, et al. ETVARD (endoscopic transanal vacuum-assisted rectal drainage) leads to complete but delayed closure of extraperitoneal rectal anastomotic leakage cavities following neoadjuvant radiochemotherapy. Int J Colorectal Dis 2009; 24: 819-25.

33. Nagell CF, Holte K. Treatment of anastomotic leakage after rectal resection with transrectal vacuum-assisted drainage (VAC). A method for rapid control of pelvic sepsis and healing. Int J Colorectal Dis 2006; 21: 657-60.

34. Arezzo A, Miegge A, Garbarini A, Morino M. Endoluminal vacuum therapy for anastomotic leaks after rectal surgery. Tech Coloproctol 2010; 14: 279-81.

35. Richterich JP, Heigl A, Muff B, et al. Endo-SPONGE: a new endoscopic treatment option in colonoscopy. Gastrointest Endosc 2008; 68: 1019-22.

36. Mees ST, Palmes D, Mennigen R, et al. Endo-vacuum assisted closure treatment for rectal anastomotic insufficiency. Dis CoIon Rectum 2008; 51: 404-10.

37. Glitsch A, von Bernstorff W, Seltrecht U, et al. Endoscopic transanal vacuum-assisted rectal drainage (ETVARD): an optimized therapy for major leaks from extraperitoneal rectal anastomoses. Endoscopy 2008; 40: 192-9.

38. Van Koperen PJ, Van Berge Henegouwen MI, Slors JF, Bemelman WA. Endo-sponge treatment of anastomotic leakage after ileo-anal pouch anastomosis: report of two cases. Colorectal Dis 2008; 10: 943-4.

39. van Koperen PJ, van Berge Henegouwen MI, Rosman C, et al The Dutch multicenter experience of the endo-sponge treatment for anastomotic leakage after colorectal surgery. Surg Endosc 2009; 23: 1379-83.

40. Riss S, Stift A, Meier M, et al. Endo-sponge assisted treatment of anastomotic leakage following colorectal surgery. Colorectal Dis 2010; 12: e104-8.

41. Riss S, Stift A, Kienbacher C, et al. Recurrent abscess after primary successful endo-sponge treatment of anastomotic leakage following rectal surgery. World I Gastroenterol 2010; 16: 4570-4.

42. Hoogenboom FJ, Hoff C, Koopal SA. Small intestinal-colorectal anastomotic fistula developing during endo-sponge treatment. Colorectal Dis 2010; 12: e337-8.

43. Einenkel J, Holler B, Hoffmeister A. Sonographic diagnosis and Endo-SPONGE assisted vacuum therapy of anastomotic leakage following posterior pelvic exenteration for ovarian cancer without using a protective stoma. J Gynecol Oncol 2011; 22 131-4.

44. Rosenberger LH, Shada A, Ritter LA, et al. Delayed endoluminal vacuum therapy for rectal anastomotic leaks after rectal resection in a swine model: a new treatment option. Clin Transl Sci 2014; 7: 121-6.

45. Wedemeyer J, Kubicka S, Lankisch TO, et al. Transgastrically placed endoscopic vacuum-assisted closure system as an addition to transgastric necrosectomy in necrotizing pancreatitis (with video). Gastrointest Endosc 2012; 76: 1238-41.

46. D'Hondt M, De Hondt G, Malisse P, et al. Chronic pelvic abscedation after completion proctectomy in an irradiated pelvis: another indication for ENDO-sponge treatment? Tech Coloproctol 2009; 13: 311-4.
47. Wallstabe I, Tiedemann A, Schiefke I. Endoscopic vacuum-assisted therapy of an infected pancreatic pseudocyst. Endoscopy 2011; 43 Suppl 2 UCTN: E312-3.

48. Wallstabe I, Tiedemann A, Schiefke I. Endoscopic vacuum-assisted therapy of infected pancreatic pseudocyst using a coated sponge. Endoscopy 2012; 44 Suppl 2 UCTN: E49-50.

49. Seyfried F, Reimer S, Miras AD, et al. Successful treatment of a gastric leak after bariatric surgery using endoluminal vacuum therapy. Endoscopy 2013; 45 Suppl 2 UCTN: E267-8.

50. Schorsch T, Muller C, Loske G. Pancreatico-gastric anastomotic insufficiency successfully treated with endoscopic vacuum therapy. Endoscopy 2013; 45 Suppl 2 UCTN: E141-2.

51. Fischer A, Richter-Schrag HJ, Hoeppner J, et al. Endoscopic intracavitary pull-through vacuum treatment of an insufficient pancreaticogastrostomy. Endoscopy 2014; 46 Suppl 1 UCTN: E218-9.

Received: 3.11.2014, accepted: 8.01.2015. 